\title{
Características clínicas y gravedad de COVID-19 en adultos
} mexicanos

\author{
Miguel A. Pérez-Sastré, ${ }^{1}$ Jesús Valdés ${ }^{2}$ y Luis Ortiz-Hernández ${ }^{3 *}$
}

${ }^{1}$ Universidad Nacional Autónoma de México, Programa de Maestría y Doctorado en Ciencias Médicas, Odontológicas y de la Salud; ${ }^{2}$ nstituto Politécnico Nacional, Departamento de Bioquímica, Centro de Investigación y de Estudios Avanzados; ${ }^{3}$ Universidad Autónoma Metropolitana Unidad Xochimilco, Departamento de Atención a la Salud. Ciudad de México, México

\section{Resumen}

Introducción: Se ha señalado que factores como obesidad, diabetes, hipertensión y edad influyen en la progresión de COVID-19; sin embargo, la evidencia para otras condiciones no es concluyente. Objetivo: Identificar qué antecedentes clínicos están relacionados con la gravedad de COVID-19 y si la edad funge como un modificador de efecto de la relación entre comorbilidades cardiometabólicas (CCM) y progresión de COVID-19. Método: Se analizaron los datos de casos confirmados $\geq 20$ años $(n=155$ 017). La hospitalización, el desarrollo de neumonía, el requerimiento de intubación, el ingreso a la unidad de cuidados intensivos y la muerte constituyeron las variables dependientes en la estimación de modelos de regresión de Poisson y la interacción entre edad y CCM, las independientes. Resultados: Tener CCM, así como otras comorbilidades, se relacionó directamente con la progresión de COVID-19. El riesgo de gravedad de COVID-19 asociado a las CCM fue menor conforme la edad era mayor. El asma y el tabaquismo no fueron factores de riesgo para la progresión de COVID-19. Conclusión: En la población mexicana, el riesgo de progresión de COVID-19 asociada a comorbilidades fue mayor en los adultos jóvenes.

PALABRAS CLAVE: Obesidad. Hipertensión. Diabetes. Asma. Tabaquismo. Infección por coronavirus. Edad. Adulto joven.

\section{Clinical characteristics and severity of COVID-19 among Mexican adults}

\begin{abstract}
Introduction: Obesity, diabetes, hypertension and age have been pointed at as factors that influence on the progression of COVID-19; however, evidence for other conditions is inconclusive. Objective: To identify which clinical characteristics are related to COVID-19 severity and to determine whether age acts as a modifier of the relationship between cardio-metabolic comorbidities (CMC) and COVID-19 progression. Method: Data on $\geq 20$-year-old confirmed cases $(n=155017)$ were analyzed. Hospitalization, development of pneumonia, intubation requirement, intensive care unit admission and death were the dependent variables in Poisson regression models estimation, whereas the interaction between age and different CMCs were the independent variables. Results: Having CMCs, as well as other comorbidities, was directly related to COVID-19 progression. The risk for COVID-19 severity was lower as age was more advanced. Asthma and smoking were not risk factors for the progression of COVID-19. Conclusion: In the Mexican population, the risk of COVID-19 progression associated with comorbidities was higher in young adults.
\end{abstract}

KEY WORDS: Obesity. Hypertension. Diabetes. Asthma. Smoking. Coronavirus infection. Age. Young adult.

Correspondencia:

*Luis Ortiz-Hernández

E-mail: lortiz@correo.xoc.uam.mx CC BY-NC-ND (http://creativecommons.org/licenses/by-nc-nd/4.0/).
Fecha de recepción: 24-06-2020

Fecha de aceptación: 23-07-2020

DOI: $10.24875 / G M M .20000430$
Gac Med Mex. 2020;156:379-387

Disponible en PubMed

www.gacetamedicademexico.com 


\section{Introducción}

El 30 de enero de 2020, la Organización Mundial de la Salud declaró la pandemia de la infección por el nuevo coronavirus SARS-CoV-2 como una emergencia de salud pública de importancia internacional. Para inicios de mayo existían millones de infectados y miles de fallecimientos. En México, para el 21 de junio existían 180545 casos de infección y 21825 personas habían fallecido por la enfermedad ocasionada por este coronavirus (COVID-19). ${ }^{1}$

La investigación se ha enfocado en determinar qué antecedentes personales y médicos de las personas infectadas influyen en la progresión de COVID-19. La obesidad, la diabetes, la hipertensión y la edad han sido señaladas como factores que influyen en el progreso de la enfermedad; ${ }^{1-3}$ sin embargo, la evidencia para otras condiciones no es concluyente, por ejemplo, los resultados respecto a la enfermedad renal crónica, la enfermedad pulmonar obstructiva crónica (EPOC), el asma ${ }^{2,4,5}$ y el tabaquismo ${ }^{6-10}$ han sido inconsistentes. Se requieren más investigaciones con menos limitaciones, ya que los primeros estudios se basaron en muestras reducidas de personas hospitalizadas y no se realizaron ajustes por variables de confusión. ${ }^{2,8,9,11}$

Se ha reconocido que el comportamiento de COVID19 adopta características específicas de acuerdo con el estado de salud de las poblaciones. Se ha especulado que el grado de afectación por COVID-19 en la población mexicana se debe a la elevada prevalencia de obesidad y otras enfermedades crónicas. En la población mexicana, la obesidad es más prevalente en los adultos jóvenes en comparación con los adultos mayores. ${ }^{12}$ En 2016, las prevalencias de hipertensión y diabetes en adultos mexicanos fueron de $31.5 \mathrm{y}$ $12.9 \%$, respectivamente. ${ }^{13}$ Considerando lo anterior, los objetivos del presente estudio fueron identificar qué antecedentes clínicos están relacionados con COVID-19 y determinar si la edad funge como un modificador de efecto de la relación entre comorbilidades cardiometabólicas y la progresión de COVID-19.

\section{Método}

Con el fin de alcanzar los objetivos del estudio, se utilizaron los datos registrados por la Dirección General de Epidemiología de la Secretaría de Salud, Gobierno Federal de México, respecto a los casos sospechosos y confirmados de infección por SARSCoV-2. ${ }^{14} \mathrm{La}$ base de datos concentra información de los casos sospechosos a los cuales se les realizó la prueba de detección, notificados ante la autoridad sanitaria en México hasta el 21 de junio de 2020, distinguiendo entre casos positivos, negativos y con resultado pendiente. La base de datos recoge información de los casos reportados por cualquier establecimiento médico del país. El 28 de febrero se notificó el primer caso en México. Una segunda fuente de información de la base de datos son los casos detectados mediante el Sistema de Vigilancia Epidemiológica de Enfermedad Respiratoria Viral (SISVER), el cual comenzó a operar el 5 de abril del año en curso. $^{15}$ El SISVER trabaja mediante 475 unidades de salud monitoras de enfermedades respiratorias (USMER), distribuidas en todas las entidades federativas. En esas unidades se toman muestras de $10 \%$ de los casos ambulatorios de enfermedad respiratoria viral y de $100 \%$ de los casos graves de la misma; las muestras recolectadas son enviadas a laboratorios de referencia para identificar la presencia de 14 virus, incluidos influenza y SARS-CoV-2.

El Comité de Ética en Investigación del Centro de Investigaciones y Estudios Avanzados aprobó los aspectos éticos del estudio. La base de datos analizada no contiene datos personales que revelen la identidad de las personas. Se utilizó un código aleatorio para identificarlos, lo que garantizó la confidencialidad de la información.

En la base de datos original se disponía de 479528 registros de todos los casos de infección respiratoria. El primer caso de infección por SARS-CoV-2 inició con síntomas el 6 de enero, por lo cual los registros previos a esa fecha fueron descartados $(n=103)$. En la Tabla 1 se presenta la distribución de los registros con datos faltantes; solo se consideraron los casos confirmados de infección por SARS-CoV-2 ( $n=159$ 660). En $96 \%$ de los casos con resultado positivo se dispuso de datos completos. Se excluyeron los menores de 20 años, por lo que la muestra analítica fue de 155017.

Como indicadores de severidad de COVID-19 se incluyeron cinco posibles desenlaces: hospitalización, desarrollo de neumonía, requerimiento de intubación, ingreso a la unidad de cuidados intensivos (UCl) y muerte.

El sexo y la edad fueron las características sociodemográficas estudiadas. La edad se categorizó en 20 a 39, 40 a 59 y mayores de 60 años. También se 
Tabla 1. Distribución de datos faltantes de acuerdo con el resultado de la prueba de PCR para identificar infección por SARS-CoV-2. México, 2020

\begin{tabular}{|c|c|c|c|c|c|}
\hline \multirow[t]{3}{*}{ Información faltante } & \multicolumn{4}{|c|}{ Resultado en prueba para SARS-CoV-2 } & \multirow[t]{3}{*}{$p$} \\
\hline & Total $(n=77005)$ & Positivo $(n=16752)$ & Negativo ( $n=49033$ ) & Pendiente $(n=11220)$ & \\
\hline & $\%$ & $\%$ & $\%$ & $\%$ & \\
\hline Etnicidad (ser indígena) & 3.11 & 2.72 & 3.32 & 3.95 & 0.000 \\
\hline Sector de la institución de salud & 0.60 & 0.61 & 0.58 & 0.68 & 0.140 \\
\hline Marginación municipal & 0.02 & 0.03 & 0.02 & 0.01 & 0.003 \\
\hline Tamaño del municipio & 0.02 & 0.03 & 0.02 & 0.02 & 0.003 \\
\hline Comorbilidades & 0.46 & 0.57 & 0.34 & 0.76 & 0.000 \\
\hline Asma & 0.33 & 0.41 & 0.24 & 0.61 & 0.000 \\
\hline Enfermedades cardiovasculares & 0.34 & 0.43 & 0.24 & 0.60 & 0.000 \\
\hline EPOC & 0.33 & 0.40 & 0.24 & 0.58 & 0.000 \\
\hline Insuficiencia renal e inmunodepresión & 0.39 & 0.48 & 0.31 & 0.62 & 0.000 \\
\hline Tabaquismo & 0.35 & 0.44 & 0.25 & 0.59 & 0.000 \\
\hline Neumonía & 0.00 & 0.00 & 0.00 & 0.00 & 0.331 \\
\hline Al menos en una variable & 4.37 & 4.05 & 4.35 & 5.46 & 0.000 \\
\hline
\end{tabular}

Tabla 2. Análisis factorial exploratorio de condiciones médicas en población con infección por SARS-CoV-2. México, 2020

\begin{tabular}{|l|c|c|c|}
\hline Variable & F1 & F2 & F3 \\
\hline Valor propio & 1.68 & 1.23 & 1.06 \\
\hline Varianza (\%) & 21.0 & 15.4 & 13.3 \\
\hline $\begin{array}{l}\text { Condición médica } \\
\text { Obesidad }\end{array}$ & & & \\
\hline Hipertensión arterial & 0.55 & -0.41 & 0.34 \\
\hline Diabetes mellitus & 0.78 & 0.01 & -0.06 \\
\hline Asma & 0.75 & -0.01 & -0.14 \\
\hline Enfermedad cardiaca & -0.14 & 0.06 & 0.86 \\
\hline EPOC & 0.26 & 0.35 & 0.14 \\
Inmunosupresión & 0.14 & 0.35 & 0.30 \\
Insuficiencia renal crónica & -0.15 & 0.72 & 0.11 \\
\hline EPOC = enfermedad pulmonar obstructiva crónica. & 0.31 & 0.48 & -0.22 \\
\hline
\end{tabular}

creó una variable en la que se expresó la edad en décadas (2, 20 a 29 años; 3, 30 a 39 años...).

En el SISVER se registran las siguientes condiciones médicas: obesidad, hipertensión arterial, diabetes mellitus, asma, enfermedades cardiacas, inmunosupresión, insuficiencia renal crónica (IRC) y EPOC. En un análisis factorial exploratorio (Tabla 2) se observó que quedaron estratificadas en dos grupos: comorbilidades cardiometabólicas y otras comorbilidades. El asma se analizó por separado y también se consideró el tabaquismo.

Las instituciones en las cuales se registró el caso fueron clasificadas en seguridad social, población abierta y servicios privados. Se calculó el número de días transcurridos entre el inicio de los síntomas y la atención recibida en los establecimientos de salud. También se estimó el número de días desde el inicio de los síntomas hasta el día del corte del reporte (21 de junio de 2020). Los estados fueron clasificados para identificar cuatro regiones: centro, norte, sur y occidente.

Para medir la posición socioeconómica de los individuos, la base de datos del SISVER se vinculó a la base de datos del Índice de Marginación Municipal de $2015,{ }^{16}$ de la cual también se obtuvo la información del tamaño de población de los municipios.

El programa Stata versión 15 se utilizó para llevar acabo el análisis estadístico. Se obtuvieron frecuencias relativas para las variables categóricas, mientras que para las variables continuas se calcularon medias. Se utilizó la prueba de chi cuadrada para identificar si existían diferencias entre las diferentes categorías. Para las variables continuas, las diferencias se buscaron por medio de $\mathrm{t}$ de Student y Anova, de 
acuerdo con el número de categorías de la variable independiente. Se estimó la prevalencia de los desenlaces de acuerdo con las características sociodemográficas y médicas de los casos.

Se estimaron modelos de regresión de Poisson de cada indicador de progresión de la enfermedad, en los cuales las características sociodemográficas y los antecedentes médicos de los pacientes constituyeron las variables independientes. En un primer modelo se estimaron los efectos principales de todas las variables. En un segundo modelo se incluyó la interacción de la edad con las comorbilidades cardiometabólicas. A partir de estos modelos se estimaron razones de prevalencias con sus respectivos intervalos de confianza de $95 \%$. Estos modelos se ajustaron por características sociodemográficas y clínicas.

\section{Resultados}

La mitad de los registros en la base de datos correspondieron a hombres y las edades más frecuentes fueron de 40 a 59 años (Tabla 3). La condición médica de mayor prevalencia fue la hipertensión arterial, seguida de obesidad y diabetes. La prevalencia de tabaquismo fue de $8 \%$. Alrededor de $30 \%$ requirió ingreso al hospital, una cuarta parte desarrolló neumonía y $13 \%$ falleció. Cerca de la mitad de los casos con resultado positivo fue atendido en una USMER. EI tiempo promedio para recibir atención médica fue de 4.2 días.

Las prevalencias de los cinco indicadores de gravedad de COVID-19 fueron más altas en los varones, en los de mayor edad, en quienes tenían más comorbilidades cardiometabólicas, en quienes tenían otras comorbilidades, cuyos casos fueron reportados en una USMER y en los del tercil más alto de tiempo transcurrido para recibir atención médica (Tabla 4). Los pacientes con asma tuvieron menor prevalencia de hospitalizaciones, neumonías, intubaciones y defunciones en comparación con quienes no padecían asma. Los fumadores tuvieron mayor proporción de hospitalizaciones, neumonías y defunciones respecto a los no fumadores. El porcentaje de hospitalizaciones fue mayor en el tercil 2 de tiempo de inicio de síntomas, mientras que en el resto de los indicadores fue mayor en el tercil 3.

En los modelos solo con efectos principales se observó que conforme la edad fue mayor o se tenían más comorbilidades cardiometabólicas, la probabilidad de presentar los indicadores de etapas severas
Tabla 3. Características de adultos mexicanos positivos a infección por SARS-CoV-2

\begin{tabular}{|c|c|}
\hline Característica & $\%$ \\
\hline Hombres & 52.2 \\
\hline \multicolumn{2}{|l|}{ Edad (años) } \\
\hline$<20$ & 2.9 \\
\hline 20-39 & 34.8 \\
\hline $40-59$ & 41.1 \\
\hline$\geq 60$ & 20.5 \\
\hline Obesidad & 19.8 \\
\hline Hipertensión arterial & 20.5 \\
\hline Diabetes mellitus & 16.8 \\
\hline \multicolumn{2}{|l|}{ CCM } \\
\hline Ninguna & 60.1 \\
\hline Una & 25.6 \\
\hline Dos o tres & 14.3 \\
\hline Enfermedades cardiacas & 2.5 \\
\hline EPOC & 1.8 \\
\hline Inmunosupresión & 1.4 \\
\hline Insuficiencia renal crónica & 2.3 \\
\hline Asma & 2.8 \\
\hline Tabaquismo & 8.0 \\
\hline Hospitalización & 32.5 \\
\hline Neumonía & 25.2 \\
\hline Intubación & 2.9 \\
\hline Ingreso a UCl & 2.8 \\
\hline Defunción & 13.0 \\
\hline \multirow[t]{2}{*}{ Casos captados en USMER } & 39.9 \\
\hline & Media \\
\hline Tiempo para atención & 4.2 \\
\hline Tiempo inicio de síntomas & 3.6 \\
\hline \multicolumn{2}{|c|}{$\begin{array}{l}\text { CCM = comorbilidad cardiometabólica, EPOC = enfermedad pulmonar obstructiva } \\
\text { crónica, USMER = unidades de salud monitoras de enfermedades respiratorias. } \\
\text { Tiempo para atención = número de días entre el desarrollo de síntomas y la atención en } \\
\text { el establecimiento de salud. } \\
\text { Tiempo inicio de síntomas = número de días entre el desarrollo de síntomas y el cierre } \\
\text { del registro ( } 21 \text { de junio, 2020). }\end{array}$} \\
\hline
\end{tabular}

de COVID-19 fue más alta (Tabla 5 y Figura 1). Tener dos o tres comorbilidades tuvo la razón de prevalencia (RP) más alta en todos los desenlaces. En general, la interacción entre edad y número de comorbilidades fue significativa, con razón de prevalencia más baja conforme la edad fue mayor, lo cual implica que la asociación entre las comorbilidades cardiometabólicas y los indicadores de mayor severidad de COVID-19 fue más fuerte en los más 


\begin{tabular}{|c|c|c|c|c|c|}
\hline \multirow[t]{2}{*}{ Característica } & Hospitalización & Neumonía & Intubación & UCI & Defunción \\
\hline & $\%$ & $\%$ & $\%$ & $\%$ & $\%$ \\
\hline $\begin{array}{l}\text { Sexo } \\
\text { Mujer } \\
\text { Hombre }\end{array}$ & $\begin{array}{c}27.4^{\ddagger} \\
37.6\end{array}$ & $\begin{array}{c}21.3^{\ddagger} \\
29.3\end{array}$ & $\begin{array}{l}2.1^{\ddagger} \\
3.7\end{array}$ & $\begin{array}{l}2.0^{\ddagger} \\
3.5\end{array}$ & $\begin{array}{c}10.1^{\ddagger} \\
15.9\end{array}$ \\
\hline 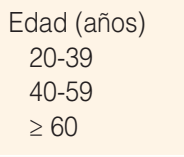 & $\begin{array}{l}13.5^{\ddagger} \\
34.4 \\
63.6\end{array}$ & $\begin{array}{l}11.0^{\ddagger} \\
26.7 \\
49.0\end{array}$ & $\begin{array}{l}0.8^{\ddagger} \\
3.1 \\
6.5\end{array}$ & $\begin{array}{l}1.0^{\ddagger} \\
2.9 \\
5.8\end{array}$ & $\begin{array}{l}2.4^{\ddagger} \\
12.0 \\
34.5\end{array}$ \\
\hline $\begin{array}{l}\text { Comorbilidad c } \\
\text { Ninguna } \\
\text { Una } \\
\text { Dos o tres }\end{array}$ & $\begin{array}{l}23.3^{\ddagger} \\
41.5 \\
57.3\end{array}$ & $\begin{array}{l}17.9^{\ddagger} \\
33.0 \\
44.5\end{array}$ & $\begin{array}{l}1.8^{\ddagger} \\
4.2 \\
5.8\end{array}$ & $\begin{array}{l}1.7^{\ddagger} \\
4.0 \\
5.4\end{array}$ & $\begin{array}{l}7.5^{\ddagger} \\
17.7 \\
28.8\end{array}$ \\
\hline $\begin{array}{l}\text { Otras comorbili } \\
\text { No } \\
\text { Sí }\end{array}$ & $\begin{array}{l}31.0^{\ddagger} \\
60.8\end{array}$ & $\begin{array}{c}24.2^{\ddagger} \\
45.8\end{array}$ & $\begin{array}{l}2.8^{\ddagger} \\
5.7\end{array}$ & $\begin{array}{c}2.7^{\ddagger} \\
5.3\end{array}$ & $\begin{array}{l}11.9^{\ddagger} \\
32.3\end{array}$ \\
\hline $\begin{array}{l}\text { Asma } \\
\text { No } \\
\text { Sí }\end{array}$ & $\begin{array}{c}33.2^{\ddagger} \\
27.0\end{array}$ & $\begin{array}{c}25.9^{\dagger} \\
21.2\end{array}$ & $\begin{array}{l}3.0 \\
2.0\end{array}$ & $\begin{array}{l}2.8 \\
4.1\end{array}$ & $\begin{array}{c}13.4^{\ddagger} \\
9.8\end{array}$ \\
\hline $\begin{array}{l}\text { Tabaquismo } \\
\text { No } \\
\text { Sí }\end{array}$ & $\begin{array}{c}33.0^{\dagger} \\
34.1\end{array}$ & $\begin{array}{c}25.6^{\ddagger} \\
27.8\end{array}$ & $\begin{array}{l}3.0 \\
3.2\end{array}$ & $\begin{array}{l}2.8 \\
2.8\end{array}$ & $\begin{array}{c}13.2^{\ddagger} \\
14.5\end{array}$ \\
\hline $\begin{array}{l}\text { Fuente de datos } \\
\text { No USMER } \\
\text { USMER }\end{array}$ & $\begin{array}{c}23.0^{\ddagger} \\
48.0\end{array}$ & $\begin{array}{c}17.9^{\ddagger} \\
37.4\end{array}$ & $\begin{array}{l}1.8^{\ddagger} \\
4.7\end{array}$ & $\begin{array}{l}2.1^{\ddagger} \\
4.0\end{array}$ & $\begin{array}{l}9.0^{\ddagger} \\
19.7\end{array}$ \\
\hline $\begin{array}{l}\text { Tiempo para ate } \\
\text { Tercil } 1 \\
\text { Tercil } 2 \\
\text { Tercil } 3\end{array}$ & $\begin{array}{l}33.6^{\ddagger} \\
28.0 \\
36.0\end{array}$ & $\begin{array}{l}22.6^{\ddagger} \\
23.5 \\
29.9\end{array}$ & $\begin{array}{l}2.0^{\ddagger} \\
3.0 \\
3.8\end{array}$ & $\begin{array}{l}1.9^{\ddagger} \\
2.6 \\
3.8\end{array}$ & $\begin{array}{l}13.7^{\star} \\
11.3 \\
14.4\end{array}$ \\
\hline $\begin{array}{l}\text { Tiempo inicio de } \\
\text { Tercil } 1 \\
\text { Tercil } 2 \\
\text { Tercil } 3\end{array}$ & $\begin{array}{c}36.6^{\ddagger} \\
43.2 \\
38.5\end{array}$ & $\begin{array}{l}19.9^{\ddagger} \\
24.3 \\
32.0\end{array}$ & $\begin{array}{l}1.5^{\ddagger} \\
2.4 \\
4.8\end{array}$ & $\begin{array}{l}1.6^{\ddagger} \\
2.3 \\
4.4\end{array}$ & $\begin{array}{l}7.5^{\ddagger} \\
12.7 \\
18.5\end{array}$ \\
\hline
\end{tabular}

jóvenes y fue menor conforme se incrementaba la edad. Los mismos resultados se observaron con la interacción de la edad y otras comorbilidades (Tabla 5 y Figura 2).

Después de ajustar por otras variables, los hombres mantuvieron riesgo más alto de avanzar a etapas más severas de COVID-19. Los pacientes con otras comorbilidades tuvieron mayor probabilidad de desarrollar complicaciones por la enfermedad. Conforme el tiempo en recibir atención médica fue más alto, la razón de prevalencia de desarrollar neumonía, requerir intubación o ingresar a la UCl fue mayor, mientras que respecto a la defunción fue menor. Al aumentar el tiempo desde que iniciaron síntomas a la fecha de corte, la prevalencia de los cinco indicadores de gravedad fue más alta.

\section{Discusión}

En concordancia con la información referida en la literatura, ${ }^{1,4,5,11,17,18}$ la mayor edad y las comorbilidades cardiometabólicas estuvieron asociadas al desarrollo de formas severas de COVID-19 en los adultos mexicanos estudiados. Un hallazgo novedoso fue que 
Probabilidad de hospitalización

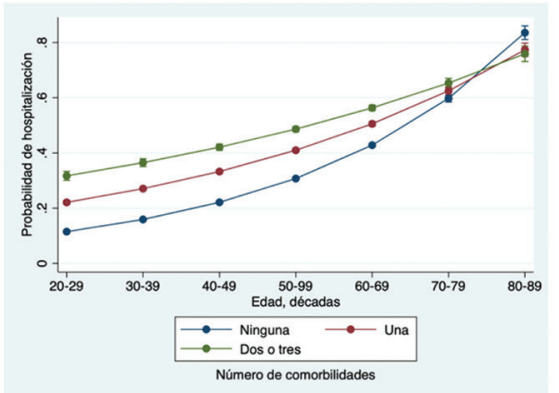

Probabilidad de intubación

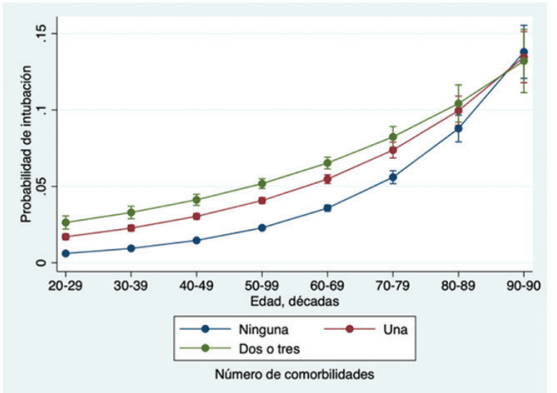

Probabilidad de muerte

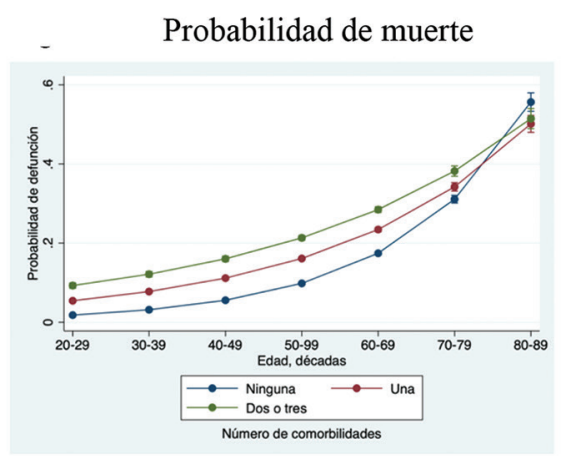

Probabilidad of neumonía

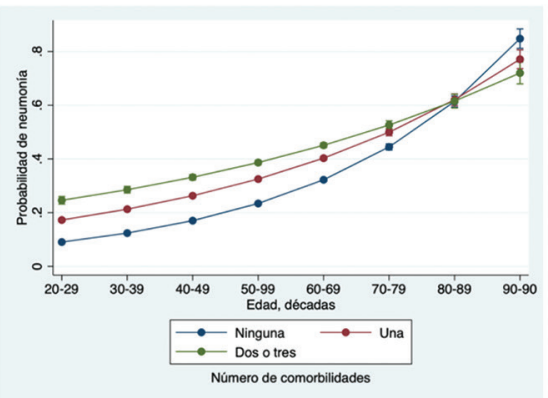

Probabilidad of admisión a

unidad de cuidados intensivos (UCI)

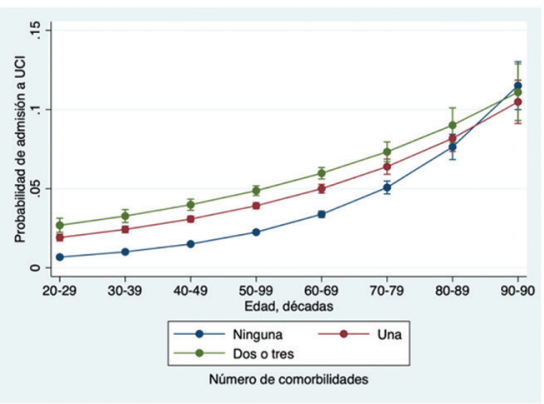

Figura 1. Probabilidades ajustadas de resultados de gravedad relacionados con COVID-19, de acuerdo con la comorbilidad cardiometabólica y la edad en adultos mexicanos con infección por SARS-CoV-2. Probabilidades ajustadas con base en los modelos reportados en la Tabla 5. En las comorbilidades se incluyen hipertensión, obesidad y diabetes.

los adultos jóvenes en México están en riesgo sustantivo de progresar a etapas severas de la infección cuando presentan comorbilidades cardiometabólicas, riesgo que disminuye conforme avanza la edad. Una posible explicación del efecto modificador de la edad es que existen diferencias entre las cohortes de edad respecto a la ganancia de peso y los cambios metabólicos que implica: considerando que la epidemia de obesidad en México inició entre las décadas de 1980 y 1990, los adultos mayores podrían haber ganado peso a edades mayores; mientras que en los adultos jóvenes, el incremento de peso pudo haberse dado incluso desde la infancia. En adultos de Noruega se observó que la ganancia de peso entre los 25 a 50 años se relacionó con accidentes cerebrovasculares; dicho riesgo no se presentó cuando la ganancia de peso ocurrió después de los 40 a 50 años. ${ }^{19}$ Esto puede indicar que la exposición a exceso de peso a edad más temprana implica mayores alteraciones orgánicas, lo que reduce la capacidad de enfrentar retos como la infección por COVID-19. También es posible que los medicamentos empleados en el tratamiento de las comorbilidades (cuyo uso puede ser más frecuente en las personas de mayor edad) actúen como factores de protección contra la progresión de la enfermedad. ${ }^{11}$

Se ha identificado que las personas con alteraciones del sistema inmunológico ${ }^{2,20,21}$ o con insuficiencia renal 
Tabla 5. Modelos de regresión de Poisson en los que las variables dependientes fueron los indicadores de progresión de COVID y las independientes, las características demográficas y antecedentes médicos. Adultos mexicanos con infección por SARS-CoV-2

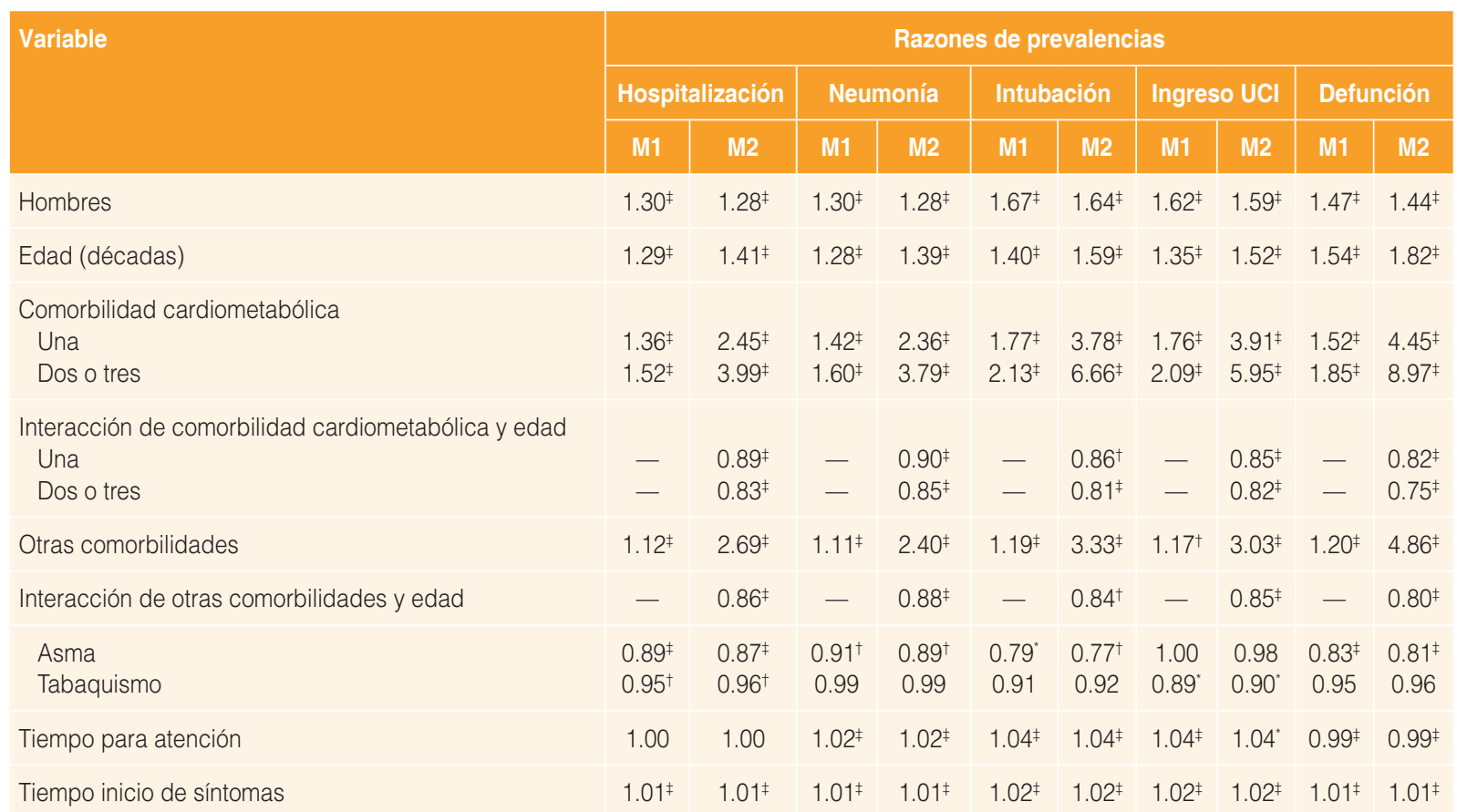

M1 = modelo solo con los efectos principales de edad y comorbilidad cardiometabólica.

M2 = modelo con la interacción de comorbilidad cardiometabólica y edad

Tiempo para atención = número de días entre el desarrollo de síntomas y la atención en el establecimiento de salud.

Tiempo inicio de síntomas = número de días entre el desarrollo de síntomas y el cierre del registro (20 de abril, 2020).

$\mathrm{UCl}=$ unidad de cuidados intensivos

$\mathrm{RP}=$ razón de prevalencias; $" p<0.05,{ }^{\dagger} p<0.01,{ }^{\ddagger} p<0.001$.

Los modelos incluyeron las variables enlistadas, además del índice de marginación y tamaño de población del municipio y la fuente de los datos.

crónica ${ }^{4,20}$ tienen mayor probabilidad de progresión de COVID-19; consistentemente se observó lo mismo en la población mexicana con estas comorbilidades. Contrario a lo que podría esperarse, el asma y tabaquismo no se relacionaron en la población mexicana con incremento de riesgo de los desenlaces, incluso se relacionaron con menor probabilidad de hospitalización en ambos casos y defunciones en el caso del asma. En otras poblaciones, el tabaquismo $0^{6,10,20}$ o las enfermedades respiratorias ${ }^{2,20}$ tampoco se han relacionado con la progresión de COVID-19. Posiblemente, las complicaciones producidas por SARS-CoV-2 se originan, sobre todo, a nivel cardiovascular y metabólico. ${ }^{22}$ Por ello, las afectaciones previas del sistema respiratorio podrían tener un papel secundario en la progresión de la enfermedad.

El mayor tiempo para recibir atención médica se relacionó positivamente con neumonía e ingreso a la $\mathrm{UCl}$, pero negativamente con defunción. Esto sugiere que el tiempo transcurrido sin recibir atención médica desde que iniciaron los síntomas influyó directamente en la aparición de complicaciones de la enfermedad.
Es probable que quienes tardaron más en recibir atención requirieron cuidados intensivos o intubación, lo cual favoreció que disminuyera el riesgo de defunción en quienes tuvieron acceso a esos recursos. Por otra parte, las personas que enfermaron al inicio de la epidemia tuvieron mayor riesgo de intubación, ingresar a la $\mathrm{UCl}$ y morir, patrón que podría explicarse, en parte, por el incremento del conocimiento en torno al tratamiento de COVID-19 entre el personal de salud, además de que se ha promovido que las personas acudan a los servicios cuando empiezan a tener síntomas que indican la progresión de la enfermedad.

A diferencia de reportes previos de muestras hospitalarias, el análisis aquí presentado se basó en los casos de infección por coronavirus confirmados en México, con lo cual aumenta la posibilidad de extrapolar los resultados. También debe reconocerse que existió un sesgo de selección, ya que en la Jornada Nacional de Sana Distancia se promovió que las personas con síntomas leves se mantuvieran en casa. Para reducir el efecto de este sesgo, en los modelos 
Probabilidad de hospitalización

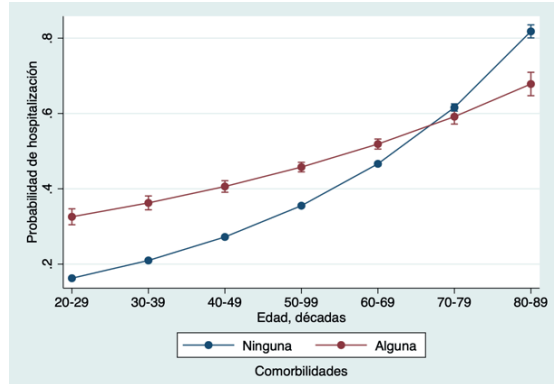

Probabilidad de intubación

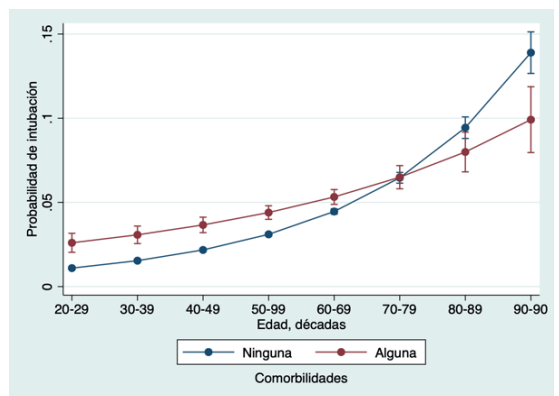

Probabilidad de muerte

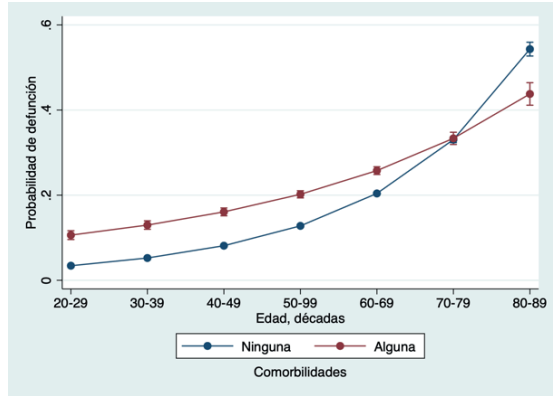

Probabilidad de neumonía

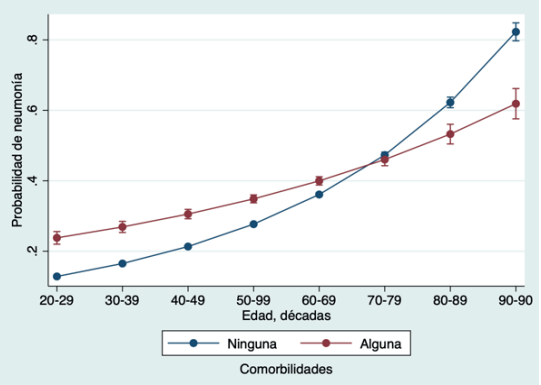

Probabilidad de admisión a unidad de cuidados intensivos (UCl)

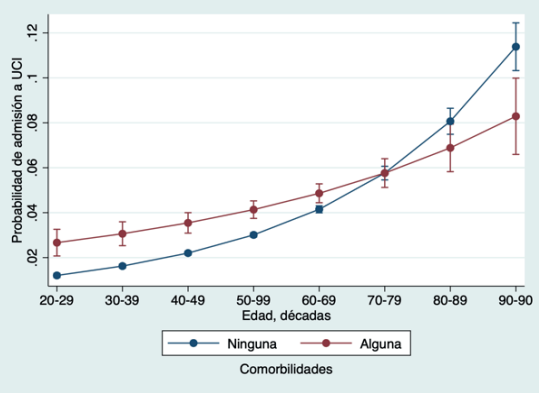

Figura 2. Probabilidades ajustadas de resultados de gravedad relacionados con COVID-19 de acuerdo con otra comorbilidad y la edad en adultos mexicanos con infección por SARS-CoV-2. Probabilidades ajustadas con base en los modelo reportados en la Tabla 5. En las comorbilidades se incluyó inmunosupresión, insuficiencia renal crónica, enfermedad cardiaca y enfermedad pulmonar obstructiva crónica.

de regresión se incluyó el tiempo entre el inicio de síntomas y el cierre del registro de casos (21 de junio), con lo cual se distinguió si los casos se presentaron al inicio o en etapas posteriores de la epidemia.

En conclusión, las enfermedades cardiometabólicas fueron las comorbilidades que con mayor fuerza se asociaron al desarrollo de complicaciones por COVID-19, seguidas de la enfermedad cardiaca, insuficiencia renal crónica, inmunosupresión y EPOC. Este resultado es preocupante dada las altas tasas de obesidad, hipertensión y diabetes que existen en México. Más aún, la edad actuó como modificador de efecto de la relación entre la presencia de comorbilidades cardiometabólicas y los indicadores de gravedad de la enfermedad. En contraste, el tabaquismo y el asma no se relacionaron con la progresión de la enfermedad, lo cual ha sido observado en otras poblaciones. Es importante descartar qué factores o condiciones no favorecen la progresión de COVID-19, ya que los esfuerzos de prevención pueden enfocarse en los grupos que realmente están en riesgo y se puede evitar generar preocupación o distrés psicológico innecesario en personas que realmente no tienen tal riesgo.

\section{Conflicto de intereses}

Los autores declaran que no tienen ningún conflicto de intereses relacionado con el manuscrito. 


\section{Financiamiento}

Los autores no recibieron ninguna subvención para llevar a cabo el manuscrito.

\section{Responsabilidades éticas}

Protección de personas y animales. Los autores declaran que para esta investigación no realizaron experimentos en seres humanos ni en animales.

Confidencialidad de los datos. Los autores declaran que siguieron los protocolos de su centro de trabajo en cuanto a la publicación de datos de pacientes.

Derecho a la privacidad y consentimiento informado. Los autores obtuvieron el consentimiento informado de los pacientes o sujetos referidos en el artículo. Este documento obra en poder del autor de correspondencia.

\section{Referencias}

1. Li B, Yang J, Zhao F, Zhi L, Wang X, Liu L, et al. Prevalence and impact of cardiovascular metabolic diseases on COVID-19 in China. Clin Res Cardiol. 2020;109:531-538.

2. Myers L, Parodi S, Escobar G, Liu V. Characteristics of hospitalized adults with COVID-19 in an integrated health care system in California. JAMA. 2020;323:2195-2198.

3. Huang $C$, Wang $Y$, Li X, Ren L, Zhao J, Hu Y, et al. Clinical features of patients infected with 2019 novel coronavirus in Wuhan, China. Lancet. 2020;395:497-506.

4. Guan WJ, Ni ZY, Hu Y, Liang WH, Ou CQ, He JX, et al. Clinical characteristics of coronavirus disease 2019 in China. N Engl J Med. 2020;382:1708-1720.

5. Zhou F, Yu T, Du R, Fan G, Liu Y, Liu Z, et al. Clinical course and risk factors for mortality of adult inpatients with COVID-19 in Wuhan, China: a retrospective cohort study. Lancet. 2020;395:1054-1062.
6. Lippi G, Henry BM. Active smoking is not associated with severity of coronavirus disease 2019 (COVID-19). Eur J Intern Med. 2020;75:107-108.

7. Guo FR. Active smoking is associated with severity of coronavirus disease 2019 (COVID-19): an update of a meta-analysis. Tob Induc Dis. 2020;18:37.

8. Vardavas CI, Nikitara K. COVID-19 and smoking: a systematic review of the evidence. Tob Induc Dis. 2020;18:20.

9. Berlin I, Thomas D, Le Faou AL, Cornuz J. COVID-19 and smoking. Nicotine Tob Res. 2020:ntaa059.

10. Rossato M, Russo L, Mazzocut S, Di Vincenzo A, Fioretto P, Vettor R. Current smoking is not associated with COVID-19. Eur Respir J. 2020;55:2001290.

11. Singh A, Gupta R, Misra A. Comorbidities in COVID-19: outcomes in hypertensive cohort and controversies with renin angiotensin system blockers. Diabetes Metab Syndr. 2020;14:283-287.

12. Barquera S, Campos-Nonato I, Hernández-Barrera L, Pedroza-Tobías A, Rivera-Dommarco J. Prevalencia de obesidad en adultos mexicanos, ENSANUT 2012. Salud Publica Mex. 2013;55.

13. Campos-Nonato I, Cuevas L, González D, Hernández-Barrera L, Shamah T, Gonzáles T, et al. Epidemiología de la obesidad y sus principales comorbilidades en México. En: Rivera-Dommarco JA, Colchero MA, Fuentes ML, González-de Cosío T, Aguilar Salinas CA, Hernández G, et al. (editores). La obesidad en México. Estado de la política pública y recomendaciones para su prevención y control. México: Instituto Nacional de Salud Pública; 2018.

14. Secretaría de Salud [Internet]. México: Datos abiertos-bases históricas: 2020.

15. Secretaría de Salud [Internet]. México: Aviso epidemiológico. Enfermedad COVID-19 por SARS-CoV-2; 2020.

16. Consejo Nacional de Población [Internet]. México: Datos abiertos del índice de marginación; 2016

17. Simonnet A, Chetboun M, Poissy J, Raverdy V, Noulette J, Duhamel A et al. High prevalence of obesity in severe acute respiratory syndrome coronavirus-2 (SARS-CoV-2) requiring invasive mechanical ventilation. Obesity (Silver Spring). 2020;28:1195-1199.

18. Lighter J, Phillips M, Hochman S, Sterling $S$, Johnson D, Francois F, et al. Obesity in patients younger than 60 years is a risk factor for $\mathrm{CO}$ VID-19 hospital admission. Clin Infect Dis. 2020;71:896-897.

19. Prestgaard E, Mariampillai J, Engeseth K, Erikssen J, Bodegard J, Liestol $\mathrm{K}$, et al. Change in body weight and long-term risk of stroke and death in healthy men. Stroke. 2020;51:1435-1441.

20. Petrilli C, Jones S, Yang J, Rajagopalan H, O'Donnell L, Chernyak Y, et al. Factors associated with hospitalization and critical illness among 4,103 patients with Covid-19 disease in New York City. medRxiv. 2020.

21. Richardson S, Hirsch J, Narasimhan M, Crawford J, McGinn T, Davidson $\mathrm{K}$, et al. Presenting characteristics, comorbidities, and outcomes among 5700 patients hospitalized with COVID-19 in the New York City area. JAMA. 2020;323:2052-2059.

22. Oxley TJ, Mock J, Majidi S, Kellner CP, Shoirah H, Singh IP, et al. Large-vessel stroke as a presenting feature of Covid-19 in the young. N Engl J Med. 2020;382:e60 